\title{
Comparison of Hand-Sewn and Stapled Anastomoses in GI Surgeries Based on Clinical Practice at Krishna Hospital, Karad
}

\author{
Dr A. Y. Kshirsagar ${ }^{1}$, Dr. Karan Singla ${ }^{2}$, Dr. Aditya Phadke ${ }^{3}$, Dr. Gayatri Bhagaria ${ }^{4}$ \\ ${ }^{1}$ Professor and Head of Unit, Department of Surgery, Krishna hospital, Karad \\ ${ }^{2}$ Resident, Department of Surgery, Krishna Hospital, Karad \\ ${ }^{3}$ Resident, Department of Surgery, Krishna Hospital, Karad \\ ${ }^{4}$ Resident, Department of Surgery, Krishna Hospital, Karad
}

\begin{abstract}
Background: There is a lack of studies comparing stapled suturing and hand-sewn suturing in GI surgeries in tier 2 towns of India. Methods: Data were retrospectively collected from 499 patients who underwent GI surgeries from January 2016 to December 2016. The patients were divided into two groups according to the method of digestive tract reconstruction: 296 patients received stapled suturing and 203 patients received hand-sewn suturing. The operation time, postoperative hospital stay, postoperative recovery and complications of the patients were evaluated and compared between the two groups. Results: The stapling procedure took shorter operative time compared to the hand-sewn procedure for gastric carcinoma, colorectal cancer and esophageal carcinoma $(P<0.05)$. There was no significant difference between the two groups in postoperative hospital stay $(P>0.05)$. Patients receiving hand sewn suturing also showed shorter recovery for gastric cancer, colorectal cancer, and shorter time to recovery of normal gastrocolorectal motility compared with patients in the stapled suturing group $(P<0.05)$ in rural areas. However, there was no difference between the two groups in terms of normal time to commencing liquid diet for esophageal cancer patients $(P>0.05)$. We also found that the stapled procedure showed a lower incidence of anastomotic leakage, anastomotic hemorrhage and stump leakage in treating colorectal cancer or gastric carcinoma compared with the hand-sewn procedure $(P<0.05)$.However the cost of staplers was too high for patients in tier 2 towns making in unaffordable for most of them. Conclusions: Application of the stapler in GI surgeries demonstrated no better effects on patients in terms of surgical operation time, recovery time to normal functions, and occurrence of complications compared to handsewn anastomosis, especially in gastric carcinoma and colorectal cancer.
\end{abstract}

Keywords: Stapler, Gastrocolorectal tumor, Anastomosis, Surgery

\section{Background}

Surgical excision was previously considered as the main method for treating gastrointestinal (GI) tumors. Anastomotic procedure is one of the key factors determining surgical success. Hand-sewn and stapled sutures comprise the major anastomotic methods in clinical practice of GI surgeries.

Staplers were originally developed to address the perceived problem of patency (security against leakage of blood or bowel contents) in anastomoses in particular [1]. After the introduction of stapled colorectal anastomosis in the 1980s, both conventional hand-sewn and stapled anastomosis have become prevalent. So far, no defined indications have been defined and most surgeons apply both techniques in clinical practice. Multiple reports reviewed available data on the trials evaluating the two techniques in colorectal surgeries and some of these results are conflicting. A systemic review evaluating 1,233 patients showed no significant difference in mortality, anastomotic leak, strictures or re-operation between stapled and hand-sewn colorectal anastomosis \{Lustosa, 2001 \#152\} [2].

However, another comprehensive review evaluating 1,125 ileo-colic participants showed stapled anastomosis was associated with fewer leaks than hand-sewn anastomosis although there was no difference for stricture forma-tion, anastomotic hemorrhage, anastomotic time, re-operation rate, mortality rate, or intra-abdominal abscess formation [3].

There has been a lack of studies comparing superiority in general surgeries of digestive tract between stapled and hand-sewn anastomosis and data based on urban patients is scant. Here we evaluated the clinical application value of the stapler suture in GI surgery for digestive tract tumors by analyzing 499 patients who were treated for various conditions of the digestive tract at nine Krishna Hospital, Karad.. We aimed to probe the differences in anastomotic effect between stapler and manual suture in treating GI tract diseases in rural areas in order to provide scientific basis for reasonable application of stapling based on clinical data.

\section{Methods}

\section{Patient Selection}

The Institutional Review Board of our hospital approved the study, which was conducted in accordance with the ethical principles of the Declaration of Helsinki and con-sistent with good clinical practices and applicable laws and regulations. All patients provided written informed consent prior to enrollment.

Patients who underwent GI surgery at Krishna hospital, 


\section{International Journal of Science and Research (IJSR) \\ ISSN (Online): 2319-7064 \\ Index Copernicus Value (2015): 78.96 | Impact Factor (2015): 6.391}

Karad in between January 2016 and December 2016, were enrolled in the study. GI tumors included gastric carcinoma, colorectal cancer and esophageal carcinoma. Exclusion criteria include: tumors with lymph node metastasis or tumors invading neighboring tissue; unresectable tumors.

\section{Research methods}

The data were collected retrospectively. All the cases were observed for one month after operation and the data relating to recovery were recorded. The follow-ing clinical characteristics were compared between the stapled and hand-sewn groups: operation time, postoperative hospital stay, postoperative recovery (time of gastrocolorectal normal motility and time to commencing normal liquid diet) and complications (anastomotic leakage, anastomotic hemorrhage, stump leakage and occurrence of emergency associated with post-surgery-ICU stay).

\section{Statistics}

Database of cases was created using Epidate3.1 (The Epidata Association, Denmark). The data of cases were processed by SPSS 19.0 software (Chicago, IL, USA). Quantitative data were expressed as $\mathrm{x} \pm \mathrm{SD}$ and discrete variables were presented using frequency table and descriptive statistical method. Comparisons between the two groups were processed using the Student's t-test, rank sum test (quantitative data) or chi-squared test (categorical data). For all the tests, $\mathrm{P}<0.05$ was con-sidered statistically significant.

\section{Results}

The stapled group consisted of 296 patients, including 187 male and 109 female patients, 239 cases from muni-cipal hospitals and 57 cases from county hospitals. The average age was $59.05 \pm 10.18$ years. The group of digest-ive tract anastomosis performed with manual procedure contained 203 cases; 136 male and 67 female patients from 165 municipal hospitals and 38 county hospitals. The average age was $57.50 \pm 10.05$ years. There were no significant difference between the two groups in gender, hospital composition and age $(\mathrm{P}>0.05)$ (gender: $\chi 2=0.769, \mathrm{P}=$ 0.38 ; hospital composition: $\chi 2=0.027, \mathrm{P}=0.88$; age: $\mathrm{t}=$ $1.65, \mathrm{P}>0.05)$. The stapler group consisted of 104 patients with gastric cancer, 128 patients of colorectal cancer and 64 patients with esophageal carcinoma respectively. The handsewn group consisted of 74 patients with gastric cancer, 97 patients with colorectal cancer and 32 patients with esophageal carcinoma respectively. Overall, there was no significant difference between the two groups in terms of the cancer composition $(\mathrm{P}>0.05)$.

Firstly we compared the operation time of gastric cancer, colorectal cancer and esophageal carcinoma in the two groups respectively (Figure 1) and the results suggest stapled anastomosis significantly shortened the operation time in the patients for the three types of tumor $(\mathrm{P}<0.05)$.

We further analyzed the number of days of postoperative hospital stay of the two groups and the results suggested no statistical significant between hand-sewn and stapled procedures for all three types of tumor $(\mathrm{P}>0.05)$ (Figure 2$)$.

To evaluate the recovery of digestive tract functions after surgery, we also compared time of recovery to normal gastrocolorectal motility after surgery between the two groups. The results revealed that the hand sewn procedure significantly shortened the time of recovery to normal functions of the digestive tract compared to the stappled procedure for gastric carcinoma and colorectal cancer $(\mathrm{P}<$ $0.05)$ but not esophageal carcinoma $(\mathrm{P}>0.05)$ (Figure 3$)$. For patients with esophageal carcinoma, we evaluated recovery time to commencing normal liquid diet after surgery and it turned out that using the stapled procedure did not alter the recovery time compared to the hand-sewn procedure.

To evaluated complications after manual and stapled anastomosis, we compared anastomotic leakage, anastomotic hemorrhage, stump leakage and the postsurgery-ICU requirement between the two groups. We found that stapler suturing showed no reduction in the incidence of anasto-motic leakage for gastric carcinoma and colorectal cancer compared to manual suturing $(\mathrm{P}<0.05)$. Stapled procedure showed no significant lowering of the incidence of anastomotic hemorrhage for the three types of digestive tract tumor $(\mathrm{P}<0.05)$. For stump leakage, the stapler suture effectively reduced the occurrence for colorectal cancer $(\mathrm{P}<0.05)$, but not for gastric carcinoma $(\mathrm{P}>0.05)$. For post-surgery-ICU requirement, there was no difference between the two groups for the three types of tumor $(\mathrm{P}>0.05)$

\section{Discussion}

GI tract diseases and tumours represent a serious threat to the health of Indian rural population . GI tumors, such as gastric cancer, liver cancer and esophageal cancer, continue to rank as the top five cancers during the past three decades in India [4]. India is one of the countries with the highest incidence of colonic cancer, which accounts for over $20 \%$ of all new gastric cancer cases in the world [5]. For esophageal cancer in India, the crude mortality rate in 2013 to 2015 was $15.2 / 100,000$, which represented $11.2 \%$ of all cancer deaths and ranked as the fourth most common cause of cancer death [6]. The incidence of colorectal cancer in India is generally lower than that in western countries, but has increased in recent years particularly in the more developed areas [7]. At present, surgery remains the major first-line treatment for these three tumors. The surgical treatment for digestive tract tumors normally involves partial or total organ removal, sur-rounding lymph node clearance and post-resection digestive tract reconstruction. Anastomosis is generally performed for the surgery treating these three types of GI tract tumor. An ideal anastomosis should fulfill the following criteria: it must be well vascularized, safe, tension-free and spillage from the operation field should be avoided [8]. The manual procedure has been used in tract anastomosis for a long time, but stapler suturing has been increasingly used as an anastomotic method in digestive tract surgery in the past few years $[9,10]$.

Our results demonstrated that stapler suturing shortened the

\section{Volume 6 Issue 1, January 2017




\section{International Journal of Science and Research (IJSR) \\ ISSN (Online): 2319-7064}

Index Copernicus Value (2015): 78.96 | Impact Factor (2015): 6.391

operation time compared to conventional hand-sewn suturing for all three types of digestive tract tumor. Shortening the operation time means reducing surgical trauma and intra-operative blood loss and also abating local infection and reducing the chance of surgical complications. However, the superiority of stapling did not extend to postoperative hospital stay, which is consistent with the previous reports that postoperative hospital stay time showed no difference between the patients who received stapler suturing and manual procedure after gastrectomy $[9,11]$. However, another study showed that stapling anastomosis shortens postoperative hospital stay in the patients with stomach and esophageal tumors [12]. This discrepancy could be due to stapling treatment and the empirical level of surgical operators.

When comparing the functional postoperative recov-ery between the two groups, the time to normal gastrocolorectal motility after surgery was shorter in the hand sewn group compared to the stappler group $(\mathrm{P}<0.05)$ indicating no increased superiority of stapplers in postoperative recovery. This could be explained by minor surgical trauma around stapler anastomosis and the contraposition of anastomotic tissues, avoiding damage to the gastric mucosa from the cutting thread $[11,13]$.

We also demonstrated that stapler suturing is not superior to the manual method in reducing the incidence of anastomotic leakage for gastric carcinoma and colorectal cancer, and the incidence of anastomotic hemorrhage for gastric carcinoma, colorectal cancer and esophageal cancer. The hand sewn suture also effectively lowered the occurrence of stump leakage for colorectal cancer com-pared to the stappler method in rural areas. Although major meta-analysis and comprehensive reviews suggest no significant difference was found in in terms of restoration of intestinal function, postoperative hospital stay and post-operative complications, it is recognized that the stapler method generally shortens the total operating time and provides better access to difficult-to-reach areas [14- 16]. We obtained similar results in operative time and hospital stay but showed no increased superiority of stapled method in reducing several complications. Consistent with our findings, a few studies did show better outcomes of stapler anastomosis in preventing occurrence of complications. For instance, stapled esophagogastric anastomosis could prevent stricture formation more effectively than hand-sewn, without increasing gastroesophageal re-flux [17, 18]. Another study also revealed the side-to-side stapled technique is conducive in decreasing complications of postoperative dysphagia and is helpful for im-proving pharyngesophageal and anastomotic menometric function [19]. Interestingly, the stapled method had a higher incidence of anastomotic stricture according to an-other report [20].

There is a relative lack of reports on the functional recovery of the organs after surgery in patients with digestive tract cancers. Our results did show that the stapler method did not significantly shorten the time to GI normal motility after surgery for both gastric and intestinal cancer patients. However, the stapler method was not superior to conventional methods in terms of the normal time to commencing liquid diet after surgery for esophageal cancer patients.

The stapled procedure could be translated to the benefits of functional recovery or reduced complications via: consistent space of anastomosis; tight closure of anastomotic nails, type "B" cross-stich using titanium nails [12]; the consistency of anastomosis and cutting may re-duce the chance of infection due to manual operation and decrease the risk of pulling and clamping the jejunum, thus reducing jejunal damage

\section{Conclusions}

Overall, our current study based on the clinical practice of Indian rural areas showed hand sewn bowel anastomosis to be superior to the stappled anastomosis method in terms of operative time, postoperative organ functional recovery time and the incidence of several com-plications, especially in gastric and colorectal cancers. Also the high cost of staplers is not affordable to patients of rural areas. The findings were somewhat inconsistent with most reported, which concluded that stapled and hand-sewn sutures had similar outcome in terms of complications. Nevertheless, our novel results indicating superiority of hand sewn anastomosis in rural areas of India in term of recovery time of gastrocolorectal normal motility suggest the hand sewn anastomosis is still a preferable choice to the stapler method in gastric and colorectal cancers given the other advantages of stapler anastomosis, such as shorter operative time.

\section{Abbreviations}

GI: gastrointestinal; S-lE: stump leakage; A-lE: anastomotic leakage; A-HH: anastomotic hemorrhage; PS-ICU: postsurgery-ICU.

\section{References}

[1] Zeebregts CJ, Heijmen RH, van den Dungen JJ, Van Schilfgaarde R: Non-suture methods of vascular anastomosis. Br J Surg 2003, 90:261-271.

[2] Lustosa SA, Matos D, Atallah AN, Castro AA: Stapled versus handsewn methods for colorectal anastomosis surgery. Cochrane Database Syst Rev 2001, CD003144.

[3] Choy PY, Bissett IP, Docherty JG, Parry BR, Merrie A, Fitzgerald A: Stapled versus handsewn methods for ileocolic anastomoses. Cochrane Database Syst Rev 2011, CD004320.

[4] Zhao P, Dai M, Chen W, Li N: Cancer trends in China. Jpn J Clin Oncol 2010, 40:281-285.

[5] $\mathrm{Bu} \mathrm{Z,} \mathrm{Ji} \mathrm{J:} \mathrm{A} \mathrm{current} \mathrm{view} \mathrm{of} \mathrm{gastric} \mathrm{cancer} \mathrm{in} \mathrm{China.}$ Transl Gastrointest Cancer 2013, 2(1):1-4.

[6] Wang JB, Fan JH, Liang H, Li J, Xiao HJ, Wei WQ, Dawsey SM, Qiao YL, Boffetta P: Attributable causes of esophageal cancer incidence and mortality in China. PLoS One 2012, 7:e42281.

[7] Xu AG, Yu ZJ, Jiang B, Wang XY, Zhong XH, Liu JH, Lou QY, Gan AH:

[8] Colorectal cancer in Guangdong Province of China: a demographic and anatomic survey. World J Gastroenterol 2010, 16:960-965. 
[9] Laukotter MG, Senninger N: Anastomotic techniques for the gastrointestinal tract. Chirurg 2013, 84:10851096. quiz 1097-1088.

[10] Korolija D: The current evidence on stapled versus hand-sewn anastomoses in the digestive tract. Minim Invasive Ther Allied Technol 2008, 17:151-154.

[11] Chunwei F, Qingzeng N, Jianliang L, Weiji W: Cervical esophagogastric anastomosis with a new stapler in the surgery of esophageal carcinoma. Eur J Cardiothorac Surg 2005, 28:291-295.

[12]Hori S, Ochiai T, Gunji Y, Hayashi H, Suzuki T: A prospective randomized trial of hand-sutured versus mechanically stapled anastomoses for gastroduodenostomy after distal gastrectomy. Gastric Cancer 2004, 7:24-30.

[13] Weijian W, Jianrong S, D L: Comparative study of complications after mechanical suture and hand suture in surgical treatment of esophageal and gastric cardiac carcinoma. J Regional Anat Operative Surg 2006, 15:76-77.

[14] Machado MA, Herman P, Montagnini AL, Eshkenazy R, Correa DS, Machado MC:

[15] An alternative linear stapling technique for performing Roux-en-Y anastomosis. Am J Surg 2002, 184:449451.

[16] Bangaru H, Veitla RM, Pigilam M, Kunwargiri GK: Comparative study between staplers and conventional (hand-sewn) anastomosis in gastrointestinal surgery. Indian J Surg 2012, 74:462-467.

[17] Chandramohan SM, Gajbhiye RN, Agwarwal A, Creedon E, Schwiers ML, Waggoner JR, Tatla D: A randomized study comparing outcomes of stapled and hand-sutured anastomoses in patients undergoing open gastrointestinal surgery. Indian J Surg 2013, 75:311316.

[18] Markar SR, Karthikesalingam A, Vyas S, Hashemi M, Winslet M: Hand-sewn versus stapled oesophagogastric anastomosis: systematic review and metaanalysis. J Gastrointest Surg 2011, 15:876-884.

[19] Wang WP, Gao Q, Wang KN, Shi H, Chen LQ: A prospective randomized controlled trial of semimechanical versus hand-sewn or circular stapled esophagogastrostomy for prevention of anastomotic stricture. World J Surg 2013, 37:1043-1050.

[20] Xu QR, Wang KN, Wang WP, Zhang K, Chen LQ: Linear stapled esophagogastrostomy is more effective than hand-sewn or circular stapler in prevention of anastomotic stricture: a comparative clinical study. J Gastrointest Surg 2011, 15:915-921.

[21]Deng B, Wang RW, Jiang YG, Tan QY, Zhao YP, Zhou JH, Liao XL, Ma Z:

[22] Functional and menometric study of side-to-side stapled anastomosis and traditional hand-sewn anastomosis in cervical esophagogastrostomy. Eur J Cardiothorac Surg 2009, 35:8-12.

[23] Luechakiettisak P, Kasetsunthorn S: Comparison of hand-sewn and stapled in esophagogastric anastomosis after esophageal cancer resection: a prospective randomized study. J Med Assoc Thai 2008, 91:681685. 